- Original Article

\title{
Association between Sleep Duration and Impaired Fasting Glucose in Korean Adults: Results from the Korean National Health and Nutrition Examination Survey 2011-2012
}

\author{
Cho-Rong Kim, Yun-Mi Song*, Jin-Young Shin, Wook Gim \\ Department of Family Medicine, Samsung Medical Center, Sungkyunkwan University School of Medicine, Seoul, Korea
}

\begin{abstract}
Background: Impaired fasting glucose (IFG) is an established risk factor for type 2 diabetes and cardiovascular disease. This study evaluated the relationship between sleep duration and IFG.

Methods: This cross-sectional study included 14,925 Korean adults (5,868 men and 9,057 women) $\geq 19$ years of age who participated in the Korean National Health and Nutrition Examination Survey between 2011 and 2012. Blood glucose levels were measured after at least eight hours of fasting. Study subjects were categorized into three groups based on self-reported sleep duration $(<7,7-8$, or $>8 \mathrm{~h} / \mathrm{d})$. IFG was diagnosed according to recommendations American Diabetes Association guidelines. Multiple logistic regression analysis was performed with adjustment for covariates.

Results: In men, short sleep duration ( $<7$ hours) was associated with increased risk of IFG (odds ratio [OR], 1.46; 95\% confidence interval [CI], 1.08 to 1.96) compared to adequate sleep duration (7-8 hours), whereas long sleep duration ( $>8$ hours) was not associated with risk of IFG (OR, 0.90 ; $95 \% \mathrm{CI}, 0.37$ to 2.18). In women, sleep duration was not associated with risk of IFG.

Conclusion: The association between sleep duration and IFG differed by sex; sleep deprivation, was associated with increased risk of IFG, especially in men.
\end{abstract}

Keywords: Koreans; Prediabetic State; Sleep; Sleep Deprivation 


\section{INTRODUCTION}

The proportion of people with type 2 diabetes has increased in recent decades, along with population aging, urbanization, obesity, and physical inactivity. The number of diabetic patients worldwide is expected to increase from 171 million in 2000 to 366 million by $2030 .{ }^{1)}$ In Korea, the prevalence of diabetes has increased by six- to seven-fold, from $1.5 \%$ to $9.9 \%$, in the past 40 years. ${ }^{2)}$ Diabetes and its associated complications such as nephropathy, neuropathy, retinopathy, and coronary and cerebral artery diseases have become a leading cause of chronic morbidity, mortality, and increased medical costs. ${ }^{3)}$

Prediabetes, including impaired fasting glucose (IFG) and impaired glucose tolerance (IGT), is a non-diabetic hyperglycemic status that does not fulfill the diagnostic criteria for diabetes, but which has clinical significance as a strong risk factor for type 2 diabetes. ${ }^{4)}$ Without specific intervention, the annual incidence rate of diabetes was $11 \%$ over a six-year period in a cohort study of Chinese adults with IFG. ${ }^{5)}$ The prediabetic state is also associated with increased cardiovascular disease and associated mortality. The risk of cardiovascular disease was more than $50 \%$ higher in prediabetes patients than in those with normal glucose tolerance, and was more evident in young adults. ${ }^{6)}$ These reports suggest the importance of controlling prediabetes, likely through lifestyle modification. ${ }^{4)}$

Sleep plays an important role in the regulation of glucose metabolism, appetite, and endocrine and immune function. ${ }^{7)}$ Inadequate sleep has been associated with obesity, ${ }^{8)}$ insulin resistance, ${ }^{9)}$ type 2 diabetes, ${ }^{10)}$ metabolic syndrome, ${ }^{11)}$ hypertension, ${ }^{8)}$ cardiovascular diseases, ${ }^{8)}$ and increased mortality. ${ }^{12)}$ Prediabetes has also been suggested to be associated with sleep duration. $^{13,14)}$

However, it is uncertain whether sleep deprivation alone or both short and oversleeping are associated with prediabetes. In addition, research on the relationship between sleep duration and prediabetes is scarce in Korea, where chronic sleep deprivation is increasingly common. ${ }^{15)}$

In this context, we conducted a cross-sectional study to evaluate the relationship between sleep duration and IFG in adult Koreans using data from the fifth Korean National Health and Nutrition Examination Survey (KNHANES V).

\section{METHODS}

\section{Study Subjects}

This study used data from the second and the third year (2011 and 2012) of the KNHANES V. The KNHANES is a nationwide cross-sectional survey designed to assess the general health and nutritional status of representative samples of Koreans, supported by the Korea Centers for Disease Control and Prevention. ${ }^{16)}$ This survey consists of health interview, health ex- amination, and nutrition surveys. ${ }^{16)}$ A total of 40,750 individuals participated in the second and the third year of the KNHANES V. Among them, 25,825 were excluded for the following reasons: < 19 years of age (9,012 people); missing data on sleep duration, fasting plasma glucose level, glycated hemoglobin (HbAlC), blood pressure, height, weight, waist circumference, or other covariates (5,471 people); diabetes mellitus defined as use of insulin or hypoglycemic agents, a fasting plasma glucose level of $126 \mathrm{mg} / \mathrm{dL}$ or higher, or HbAlC level of $6.5 \%$ or higher (3,716 people); hypertension defined as blood pressure greater than or equal to $140 / 90 \mathrm{~mm} \mathrm{Hg}$, or regular use of antihypertensive medication (6,301 people); cardiovascular disease or stroke (271 people); cancer (439 people); or depression (615 people). Finally, a total of 14,925 subjects (5,868 men and 9,057 women) were included in this study.

This study was approved by the institutional review board of the Samsung Medical Center (IRB file No. 2014-11-052). The data used for this study did not include any identifiable personal information and were publically accessible for health research. Thus, the institutional review board waived informed consent.

\section{Study Variables}

We assessed sleep duration by asking "On average, how many hours a day do you usually sleep?" through a self-administered questionnaire. Based on their responses, we categorized study subjects into three groups according to their sleep duration: short $(<7 \mathrm{~h} / \mathrm{d})$, adequate $(7-8 \mathrm{~h} / \mathrm{d})$, and long $(>8 \mathrm{~h} / \mathrm{d})$.

Venous blood was drawn after at least eight hours of fasting, and glucose levels were measured using a Hitachi Automatic Analyzer 7600 (Hitachi, Tokyo, Japan) in a qualified central laboratory. IFG was defined as fasting glucose levels between 100$125 \mathrm{mg} / \mathrm{dL}$, according to criteria from the American Diabetes Association. $^{4)}$

Level of education, monthly family income, marital status, ever-smoking, current alcohol use, physical activity, stress recognition, and menopausal status were assessed using a selfadministered questionnaire. Education level was categorized into two groups ( $<$ high school, or $\geq$ high school). Monthly family income was categorized into four groups $(\leq 1,000,000$, $1,000,001-2,000,000,2,000,001-3,000,000$, or $\geq 3,000,001$ Korean won). Marital status was categorized as ever-married or never-married. Ever-smoking was defined as having smoked at least 100 cigarettes in a lifetime. Current alcohol use was defined as at least one drink per month for the previous year. Regular physical activity was defined as participating in moderate activity for more than $30 \mathrm{~min} / \mathrm{d}$ at least five days per week, or vigorous activity for more than $20 \mathrm{~min} / \mathrm{d}$ at least three days per week. Stress recognition was assessed by asking "How much stress do you usually experience in your daily life?", and then categorized into two groups (high or low levels of stress). Menopausal status was categorized as premenopausal or postmeno- 
pausal states using a self-administered questionnaire. Body mass index (BMI, $\mathrm{kg} / \mathrm{m}^{2}$ ) was calculated using measured weight and height. BMI was categorized as underweight $\left(<18.5 \mathrm{~kg} / \mathrm{m}^{2}\right)$, normal weight $\left(18.5-22.9 \mathrm{~kg} / \mathrm{m}^{2}\right)$, or overweight/obese $(\geq 23$ $\mathrm{kg} / \mathrm{m}^{2}$ ), according to recommendations from the Korean Society for the Study of Obesity. ${ }^{17)}$

\section{Statistical Analysis}

All analyses were conducted considering the complex design and sampling weight used for the KNHANES V. Differences in the distribution of baseline characteristics among categories of sleep duration were compared by the analysis of variance or chi-square tests. To evaluate the association between IFG and sleep duration, odds ratios (ORs) and 95\% confidence intervals (CIs) were estimated by multiple logistic regression analysis after adjusting for age, sex, and selected covariates (BMI, level of education, monthly family income, marital status, ever-smoking, current alcohol use, regular physical activity, stress recognition, and menopausal status). These covariates were selected because they were significantly associated with IFG or sleep duration in our study or in previous studies..$^{10,13,14,18-20)}$ Statistical analysis was carried out using PASW SPSS Statistics for Windows ver. 18.0 (SPSS Inc., Chicago, IL, USA). P-values < 0.05 were considered statistically significant.

\section{RESULTS}

Table 1 shows the baseline characteristics of the study subjects according to sleep duration. In men, $41.4 \%$ and $5.4 \%$ were shortand long-duration sleepers, respectively. Only the distribution of regular physical activity significantly differed among the three sleep groups, and there was no difference in the distribution of other characteristics in men. In women, $37.7 \%$ and $7.5 \%$ were short- and long-duration sleepers, respectively. The distribution of age, education level, monthly family income, marital status, current alcohol use, stress level, and menopausal status were significantly different among women in the three sleep groups.

Figure 1 shows the IFG prevalence according to sleep dura-

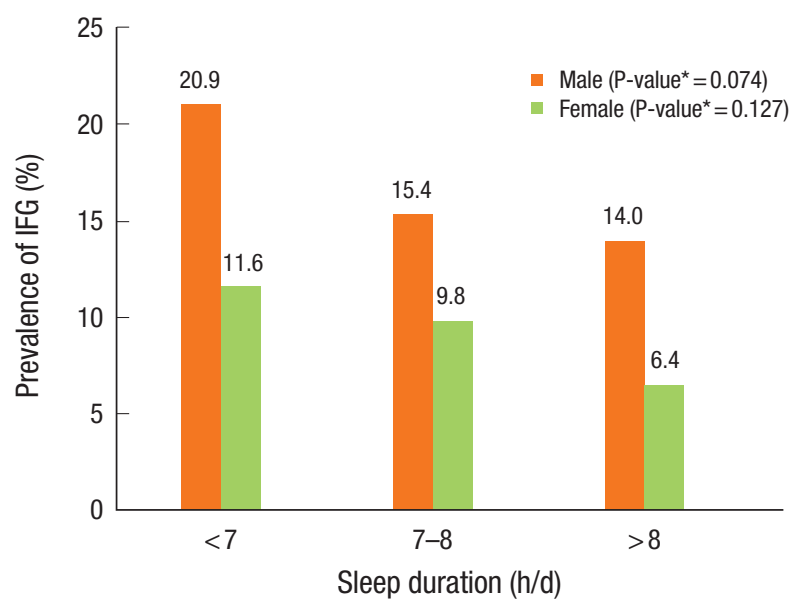

Figure 1. Prevalence of IFG according to sleep duration. IFG, impaired fasting glucose. *Obtained by chi-square test.

Table 1. Baseline characteristics of study participants by sleep duration categories

\begin{tabular}{|c|c|c|c|c|c|c|c|c|}
\hline \multirow{2}{*}{ Characteristic } & \multicolumn{4}{|c|}{ Men } & \multicolumn{4}{|c|}{ Women } \\
\hline & $<7 \mathrm{~h}$ & $7-8 \mathrm{~h}$ & $>8 \mathrm{~h}$ & P-value* & $<7 \mathrm{~h}$ & $7-8 \mathrm{~h}$ & $>8 \mathrm{~h}$ & P-value* \\
\hline Total no. of subjects & $2,431(41.4)$ & 3,123 (53.2) & $314(5.4)$ & & $3,413(37.7)$ & 4,966 (54.8) & $678(7.5)$ & \\
\hline Age $(y)$ & $39.6 \pm 0.8$ & $39.0 \pm 0.5$ & $40.6 \pm 2.1$ & 0.583 & $43.4 \pm 0.6$ & $38.8 \pm 0.4$ & $34.2 \pm 1.2$ & $<0.001$ \\
\hline $\begin{array}{l}\text { Body mass index }\left(\mathrm{kg} / \mathrm{m}^{2}\right) \\
\quad<18.5 \\
18.5-22.9 \\
\geq 23\end{array}$ & $\begin{array}{c}51(1.9) \\
975(39.8) \\
1,405(58.3)\end{array}$ & $\begin{array}{c}122(4.1) \\
1,350(42.2) \\
1,651(53.7)\end{array}$ & $\begin{array}{c}11(4.0) \\
133(34.9) \\
170(61.1)\end{array}$ & 0.167 & $\begin{array}{c}228(7.2) \\
1,620(48.8) \\
1,565(44.0)\end{array}$ & $\begin{array}{c}411(9.6) \\
2,609(51.6) \\
1,946(38.8)\end{array}$ & $\begin{array}{r}70(12.7) \\
353(49.4) \\
255(37.9)\end{array}$ & 0.162 \\
\hline Waist circumference (cm) & $82.9 \pm 0.4$ & $82.1 \pm 0.4$ & $82.9 \pm 1.1$ & 0.381 & $75.9 \pm 0.5$ & $75.1 \pm 0.4$ & $76.0 \pm 1.0$ & 0.196 \\
\hline Education level $\geq$ high school & $1,995(85.9)$ & $2,597(87.9)$ & $202(78.0)$ & 0.054 & $2,364(74.1)$ & $4,084(85.3)$ & 537 (86.8) & $<0.001$ \\
\hline $\begin{array}{l}\text { Monthly family income (Korean won) } \\
\leq 1,000,000 \\
1,000,001-2,000,000 \\
2,000,001-3,000,000 \\
\geq 3,000,001\end{array}$ & $\begin{array}{r}240(7.9) \\
357(15.3) \\
424(19.6) \\
1,410(57.3)\end{array}$ & $\begin{array}{r}309(8.7) \\
483(17.3) \\
616(20.1) \\
1,715(53.9)\end{array}$ & $\begin{array}{r}57(11.5) \\
66(25.5) \\
48(15.3) \\
143(47.7)\end{array}$ & 0.414 & $\begin{array}{r}449(10.2) \\
577(18.7) \\
614(18.8) \\
1,773(52.3)\end{array}$ & $\begin{array}{r}321(6.2) \\
708(16.0) \\
880(18.7) \\
3,057(59.0)\end{array}$ & $\begin{array}{r}85(11.2) \\
97(16.2) \\
166(23.9) \\
330(48.7)\end{array}$ & 0.015 \\
\hline Ever-married & $1,870(67.0)$ & $2,320(62.8)$ & $215(53.4)$ & 0.086 & $2,820(78.0)$ & $4,000(74.6)$ & $460(59.2)$ & $<0.001$ \\
\hline Ever-smoking ${ }^{\dagger}$ & $1,760(69.1)$ & $2,225(68.3)$ & $241(74.3)$ & 0.654 & $291(11.0)$ & $381(10.1)$ & $74(16.4)$ & 0.108 \\
\hline Current alcohol use $e^{\ddagger}$ & $1,772(74.5)$ & $2,233(73.7)$ & $213(68.3)$ & 0.605 & $1,445(44.1)$ & $2,175(46.4)$ & $345(56.7)$ & 0.020 \\
\hline Regular physical activity ${ }^{\S}$ & $485(19.9)$ & $696(24.0)$ & $75(35.9)$ & 0.025 & $564(17.1)$ & $703(14.6)$ & $86(13.8)$ & 0.354 \\
\hline Under high level of stress & $659(27.5)$ & $685(21.9)$ & $70(23.7)$ & 0.123 & $1,134(33.8)$ & $1,204(26.7)$ & $181(26.0)$ & 0.005 \\
\hline Postmenopausal state" & - & - & - & - & $1,518(35.9)$ & $1,458(22.5)$ & $206(23.4)$ & $<0.001$ \\
\hline Daily energy intake (kcal) & $2,502.4 \pm 85.5$ & $2,469.8 \pm 47.4$ & $2,368.0 \pm 284.2$ & 0.843 & $1,729.8 \pm 32.4$ & $1,728.9 \pm 20.2$ & $1,757.4 \pm 73.2$ & 0.927 \\
\hline Daily carbohydrate intake (g) & $368.2 \pm 10.7$ & $361.1 \pm 6.3$ & $341.7 \pm 30.1$ & 0.564 & $283.4 \pm 5.1$ & $276.9 \pm 3.3$ & $289.3 \pm 12.4$ & 0.281 \\
\hline
\end{tabular}

Values are presented as mean \pm standard error or number (\%).

${ }^{*}$ Obtained by analysis of variance for continuous variables or chi-square test for categorical variables. ${ }^{\dagger}$ At least 100 cigarettes in a lifetime. ${ }^{\ddagger}$ At least on drink per month for the

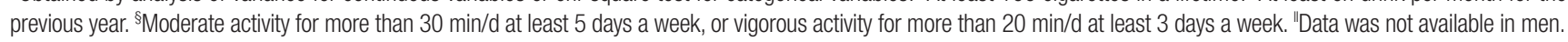


Table 2. Association between sleep duration and impaired fasting glucose

\begin{tabular}{|c|c|c|c|c|}
\hline \multirow{2}{*}{ Sleep duration } & \multicolumn{2}{|c|}{ Un-adjusted } & \multicolumn{2}{|c|}{ Multivariable-adjusted* } \\
\hline & OR $(95 \% \mathrm{Cl})$ & P-value ${ }^{\dagger}$ & OR $(95 \% \mathrm{Cl})$ & P-value \\
\hline \multicolumn{5}{|c|}{ All participants (h) } \\
\hline$<7$ & $1.38(1.11-1.73)$ & 0.004 & $1.21(0.96-1.53)$ & 0.108 \\
\hline $7-8$ & 1 & & 1 & \\
\hline$>8$ & $0.70(0.41-1.21)$ & 0.199 & $0.80(0.42-1.49)$ & 0.472 \\
\hline \multicolumn{5}{|l|}{ Men (h) } \\
\hline$<7$ & $1.46(1.08-1.96)$ & 0.013 & $1.41(1.03-1.92)$ & 0.032 \\
\hline $7-8$ & 1 & & 1 & \\
\hline$>8$ & $0.90(0.37-2.18)$ & 0.810 & $0.82(0.30-2.26)$ & 0.699 \\
\hline \multicolumn{5}{|l|}{ Women (h) } \\
\hline$<7$ & $1.22(0.89-1.65)$ & 0.215 & $0.96(0.70-1.33)$ & 0.806 \\
\hline $7-8$ & 1 & & 1 & \\
\hline$>8$ & $0.63(0.31-1.27)$ & 0.195 & $0.72(0.33-1.56)$ & 0.406 \\
\hline
\end{tabular}

$\mathrm{OR}$, odds ratio; $\mathrm{Cl}$, confidence interval.

*Adjusted for sex (in all participants), age, body mass index, education level, monthly income, marital status, cigarette smoking, current alcohol use, regular physical activity, stress recognition, and menopausal status (in women). ${ }^{\dagger}$ Obtained by multiple logistic regression analysis.

tion categories by sex. The overall IFG prevalence was higher in men $(17.6 \%)$ than in women $(10.1 \%)(\mathrm{P}<0.001)$. In men, the prevalence of IFG among short-, adequate-, and long-duration sleepers was $20.9 \%, 15.4 \%$, and $14.0 \%$, respectively. In women, the prevalence was $11.6 \%, 9.8 \%$, and $6.4 \%$, respectively. However, the prevalence of IFG was not significantly different between sleep duration categories both in men $(\mathrm{P}=0.074)$ and women $(\mathrm{P}=0.127)$.

Table 2 shows the results of multiple logistic regression analysis conducted to evaluate the association between sleep duration and IFG. The un-adjusted analysis showed that short sleep duration in men ( $<7$ hours) was associated with increased risk of IFG (OR, 1.46; 95\% CI, 1.08 to 1.96) compared to 7-8 hours of sleep. This association persisted even after adjusting for covariates (OR, 1.41; 95\% CI, 1.03 to 1.92). However, long sleep duration (>8 hours) was not associated with risk of IFG regardless of covariates adjustment. In women, shorter and longer sleep durations were not associated with risk of IFG, regardless of covariate adjustments. Subgroup analysis according to menopausal status (premenopausal or postmenopausal state) also showed no association between sleep duration and IFG, regardless of covariate adjustments.

\section{DISCUSSION}

In this large, cross-sectional study, we found that short sleep duration in men $(<7 \mathrm{~h} / \mathrm{d})$ was associated with an increased risk of IFG, compared to adequate sleep duration (7-8 h/d). However, there was no association between sleep duration and IFG in women, and long sleep duration was not associated with increased risk of IFG.

These findings on the association between short sleep duration and IFG in men are consistent with those of previous studies. Short sleep duration has consistently been shown to be as- sociated with increased risk of diabetes or prediabetes. ${ }^{10,13,14,18-20)}$ The Sleep Heart Health Study in American men and women found that self-reported short sleep duration ( $\leq 6$ hours) was associated with higher risk of diabetes or IGT compared to 7-8 hours of sleep. ${ }^{10)}$ A longitudinal (around six years of mean follow-up time) Quebec Family Study of 276 individuals aged 21 to 64 years found that an adjusted relative risk for the incidence of type 2 diabetes or IGT of 2.78 (95\% CI, 1.61 to 4.12 ) in the short sleep group ( $\leq 6$ hours), compared with the adequate sleep group (7-8 hours). ${ }^{19)}$ Short sleep duration ( $\leq 5$ hours) was also associated with increased prevalence of type 2 diabetes (OR, 2.40; 95\% CI, 1.18 to 4.91) in Korean men without abdominal obesity. ${ }^{20)}$ Lou et al. ${ }^{14)}$ reported that short sleep duration ( $<6$ hours) increased the risk of IFG in a Chinese population after adjusting for age, obesity, family history of diabetes, alcohol consumption, smoking, physical activity, and other diseases. A nested case-control study showed a three-fold increased risk of IFG (95\% CI, 1.05 to 8.59) in short-duration sleepers $(<6$ hours) compared with adequate-duration sleepers (6-8 hours), after considering several diabetes risk factors. ${ }^{13)}$ Engeda et al. ${ }^{18)}$ reported short sleep duration ( $\leq 5$ hours) to be associated with higher risk of clinically identified prediabetes.

There are several possible biological pathways through which sleep deprivation can lead to abnormal glucose regulation. First, sleep deprivation may lead to changes in the neurohormonal regulation of feeding behaviors. ${ }^{21-23)}$ A previous study examined the effect of sleep curtailment on mean levels of anorexigenic hormone (leptin) and appetite-stimulating hormone (ghrelin), hunger sense, and appetite. Mean leptin levels were $18 \%$ lower and mean ghrelin levels were $28 \%$ higher in participants who spent four hours in bed compared to their levels after 10 hours in bed. Short sleep duration (four hours) was also associated with a $24 \%$ and $23 \%$ increase in hunger and appetite, respectively, particularly for calorie-dense nutrients with high carbohydrate content such as sweets, salty snacks, and starchy food. ${ }^{21)}$ The current study compared daily energy intake and carbohydrate intake among the three sleep groups; however, there was no difference in the distribution of daily energy and carbohydrate intake among the three groups in either men or women (Table 1). Sleep restriction to $5 \mathrm{~h} / \mathrm{d}$ for one week resulted in significantly reduced insulin sensitivity in young healthy men. ${ }^{22)}$ Moreover, short sleep duration may facilitate the development of insulin resistance and reduced glucose tolerance. ${ }^{23)}$ Another possible pathway is the activation of the sympathetic nervous system. Among 11 young men with sleep restriction to $4 \mathrm{~h} / \mathrm{d}$ for six nights, evening cortisol concentrations were elevated and the activity of the sympathetic nervous system was increased. ${ }^{24)}$ The activation of inflammatory pathways may also play a role in the disruption of glucose metabolism. Reduced sleep is associated with increased tumor necrosis factor alpha, interleukin 6, and C-reactive protein. ${ }^{25)}$ Furthermore, it is well known 
that inflammatory markers are strong predictors of type 2 diabetes. $^{26)}$

Findings are inconsistent on the association between long sleep duration and diabetes or prediabetes. ${ }^{10,14,19)}$ The Sleep Heart Health Study found that self-reported long sleep duration ( $\geq 9$ hours) was associated with a higher risk of diabetes or IGT compared to 7-8 hours of sleep. ${ }^{10)}$ The Quebec Family Study found that adjusted relative risk for the incidence of type 2 diabetes or IGT was 2.54 (95\% CI, 1.42 to 3.53) for long sleep group ( $\geq 9$ hours). ${ }^{19)}$ A Chinese study found that long sleep duration (>8 hours) increased the risk of IFG. ${ }^{14)}$ However, some studies found no association between long sleep duration and the risk of diabetes or prediabetes, as shown in our study. ${ }^{13,18,20)}$ In addition, the biological mechanisms underlying the association between excessively long sleep duration and diabetes or prediabetes remain unknown. ${ }^{19)}$

IFG prevalence differs by sex, and men tend to have a higher rate of IFG than women, ${ }^{27)}$ as found in our study. Compared to women, men are known to have a lower sleep efficiency and higher wakefulness during sleep. ${ }^{28)}$ Men also tend to have a longer stage 1 sleep and shorter rapid eye movement sleep latency. ${ }^{28)}$ Thus, the association between short sleep duration and IFG may be amplified in men. Given these differences in IFG prevalence and sleep characteristics, it seems necessary to evaluate the association between IFG and sleep duration separately in men and women. Moreover, menopausal status was selected as an additional covariate in women because the postmenopausal state is significantly associated with the presence of type 2 diabetes and prediabetes. ${ }^{29)}$ We found that the association between IFG and sleep duration differs by sex. However, we could not compare the findings of our study to those of other studies because most previous studies did not separately evaluate the association by sex. Although one study reported that a significant association between limited sleep duration and metabolic syndrome in men, it did not report the relationship between sleep duration and IFG. ${ }^{11)}$

This study has several limitations. First, the cross-sectional study design prevented determination of the causal direction of the relationship between sleep duration and IFG. Second, sleep duration was assessed by self-report. Considering that assessment of sleep time by self-report was found to be moderately valid compared to measured sleep $(r=0.45)$ in a study, ${ }^{30}{ }^{2}$ self-reporting may result in a biased estimation of the association between short sleep duration and IFG. Third, we could not assess sleep quality in this study. Because poor sleep quality has been associated with the risk of diabetes or prediabetes, ${ }^{14,18)}$ it is necessary to evaluate the total effect of sleep, including quality as well as quantity, on the risk of IFG. Fourth, we could not consider the probable confounding effects of medical comorbidities, sedentary lifestyle, and dietary factors. Finally, we determined glycemic status based on a one-time measurement of fasting glucose concentration, which may have resulted in IFG misclassification.

In conclusion, the findings of this cross-sectional study of Korean adults suggest that short sleep duration rather than long sleep duration may increase the risk of IFG, although the association between short sleep duration and IFG was evident only in men.

\section{CONFLICT OF INTEREST}

No potential conflict of interest relevant to this article was reported.

\section{ACKNOWLEDGMENTS}

This research was supported by the Basic Science Research Program through the National Research Foundation of Korea funded by the Ministry of Science, ICT and Future Planning (2014R1A2A2A01002705).

\section{REFERENCES}

1. Wild S, Roglic G, Green A, Sicree R, King H. Global prevalence of diabetes: estimates for the year 2000 and projections for 2030. Diabetes Care 2004;27:1047-53.

2. Kim DJ. The epidemiology of diabetes in Korea. Diabetes Metab J 2011; 35:303-8.

3. Moon EJ, Jo YE, Park TC, Kim YK, Jung SH, Kim HJ, et al. Clinical characteristics and direct medical costs of type 2 diabetic patients. Korean Diabetes J 2008;32:358-65.

4. American Diabetes Association. Standards of medical care in diabetes--2014. Diabetes Care 2014;37 Suppl 1:S14-80.

5. Li G, Zhang P, Wang J, Gregg EW, Yang W, Gong Q, et al. The long-term effect of lifestyle interventions to prevent diabetes in the China Da Qing Diabetes Prevention Study: a 20-year follow-up study. Lancet 2008;371: 1783-9.

6. Ford ES, Zhao G, Li C. Pre-diabetes and the risk for cardiovascular disease: a systematic review of the evidence. J Am Coll Cardiol 2010;55: 1310-7.

7. Spiegel K, Leproult R, van Cauter E. Impact of sleep debt on metabolic and endocrine function. Lancet 1999;354:1435-9.

8. Buxton OM, Marcelli E. Short and long sleep are positively associated with obesity, diabetes, hypertension, and cardiovascular disease among adults in the United States. Soc Sci Med 2010;71:1027-36.

9. Spiegel K, Knutson K, Leproult R, Tasali E, van Cauter E. Sleep loss: a novel risk factor for insulin resistance and type 2 diabetes. J Appl Physiol (1985) 2005;99:2008-19.

10. Gottlieb DJ, Punjabi NM, Newman AB, Resnick HE, Redline S, Baldwin $\mathrm{CM}$, et al. Association of sleep time with diabetes mellitus and impaired glucose tolerance. Arch Intern Med 2005;165:863-7.

11. Wu MC, Yang YC, Wu JS, Wang RH, Lu FH, Chang CJ. Short sleep duration associated with a higher prevalence of metabolic syndrome in an apparently healthy population. Prev Med 2012;55:305-9. 
12. Cappuccio FP, D’Elia L, Strazzullo P, Miller MA. Sleep duration and allcause mortality: a systematic review and meta-analysis of prospective studies. Sleep 2010;33:585-92.

13. Rafalson L, Donahue RP, Stranges S, Lamonte MJ, Dmochowski J, Dorn J, et al. Short sleep duration is associated with the development of impaired fasting glucose: the Western New York Health Study. Ann Epidemiol 2010;20:883-9.

14. Lou P, Chen P, Zhang L, Zhang P, Chang G, Zhang N, et al. Interaction of sleep quality and sleep duration on impaired fasting glucose: a population-based cross-sectional survey in China. BMJ Open 2014;4:e004436.

15. Choi JH, Kim DH, Kim JY. Sleeping environment of Korean: focused on indoor condition and sleeping habits. J Korean Soc Living Environ Sys 2008;15:85-91.

16. Ministry of Health and Welfare, Korea Centers for Disease Control and Prevention. The fifth Korea National Health and Nutrition Examination Survey guide book (KNHANES V 2010-2012). Cheongju: Korea Centers for Disease Control and Prevention; 2012.

17. Kim MK, Lee WY, Kang JH, Kang JH, Kim BT, Kim SM, et al. 2014 clinical practice guidelines for overweight and obesity in Korea. Endocrinol Metab (Seoul) 2014;29:405-9.

18. Engeda J, Mezuk B, Ratliff S, Ning Y. Association between duration and quality of sleep and the risk of pre-diabetes: evidence from NHANES. Diabet Med 2013;30:676-80.

19. Chaput JP, Despres JP, Bouchard C, Astrup A, Tremblay A. Sleep duration as a risk factor for the development of type 2 diabetes or impaired glucose tolerance: analyses of the Quebec Family Study. Sleep Med 2009;10:919-24.

20. Kim J, Kim HM, Kim KM, Kim DJ. The association of sleep duration and type 2 diabetes in Korean male adults with abdominal obesity: the Korean National Health and Nutrition Examination Survey 2005. Diabetes Res Clin Pract 2009;86:e34-6.

21. Spiegel K, Tasali E, Penev P, van Cauter E. Brief communication: sleep curtailment in healthy young men is associated with decreased leptin levels, elevated ghrelin levels, and increased hunger and appetite. Ann Intern Med 2004;141:846-50.

22. Buxton OM, Pavlova M, Reid EW, Wang W, Simonson DC, Adler GK. Sleep restriction for 1 week reduces insulin sensitivity in healthy men. Diabetes 2010;59:2126-33.

23. Nedeltcheva AV, Kessler L, Imperial J, Penev PD. Exposure to recurrent sleep restriction in the setting of high caloric intake and physical inactivity results in increased insulin resistance and reduced glucose tolerance. J Clin Endocrinol Metab 2009;94:3242-50.

24. Leproult R, Copinschi G, Buxton O, van Cauter E. Sleep loss results in an elevation of cortisol levels the next evening. Sleep 1997;20:865-70.

25. Irwin MR, Wang M, Campomayor CO, Collado-Hidalgo A, Cole S. Sleep deprivation and activation of morning levels of cellular and genomic markers of inflammation. Arch Intern Med 2006;166:1756-62.

26. Pradhan AD, Manson JE, Rifai N, Buring JE, Ridker PM. C-reactive protein, interleukin 6 , and risk of developing type 2 diabetes mellitus. JAMA 2001;286:327-34.

27. Liu S, Wang W, Zhang J, He Y, Yao C, Zeng Z, et al. Prevalence of diabetes and impaired fasting glucose in Chinese adults, China National Nutrition and Health Survey, 2002. Prev Chronic Dis 2011;8:A13.

28. Roehrs T, Kapke A, Roth T, Breslau N. Sex differences in the polysomnographic sleep of young adults: a community-based study. Sleep Med 2006;7:49-53.

29. Heianza Y, Arase Y, Kodama S, Hsieh SD, Tsuji H, Saito K, et al. Effect of postmenopausal status and age at menopause on type 2 diabetes and prediabetes in Japanese individuals: Toranomon Hospital Health Management Center Study 17 (TOPICS 17). Diabetes Care 2013;36:4007-14.

30. Lauderdale DS, Knutson KL, Yan LL, Liu K, Rathouz PJ. Self-reported and measured sleep duration: how similar are they? Epidemiology 2008; 19:838-45 